Ger J Exerc Sport Res 2021 · 51:506-517 https://doi.org/10.1007/s12662-021-00760-4 Received: 13 April 2021

Accepted: 15 September 2021

Published online: 14 October 2021

(c) The Author(s) 2021

\author{
Jelena Bezold ${ }^{1}$ (D) - Sandra Trautwein ${ }^{1}$ Bettina Barisch-Fritz ' Andrea Scharpf ' \\ Janina Krell-Roesch ${ }^{1,2} \cdot$ Claudio R. Nigg $^{3} \cdot$ Alexander Woll $^{1}$ \\ ${ }^{1}$ Institute of Sports and Sports Science, Karlsruhe Institute of Technology, Karlsruhe, Germany \\ ${ }^{2}$ Department of Quantitative Health Sciences, Mayo Clinic, Rochester, USA \\ ${ }^{3}$ Institute of Sport Science, University of Bern, Bern, Switzerland
}

\title{
Effects of a 16-week multimodal exercise program on activities of daily living in institutionalized individuals with dementia
}

\section{A multicenter randomized controlled trial}

\section{Supplementary Information \\ The online version of this article (https://doi. org/10.1007/s12662-021-00760-4) contains supplementary material, which is available to authorized users.}

\section{Background}

To date, more than 50 million people worldwide are living with dementia, and by the end of 2050, nearly 152 million individuals with dementia (IWD) are expected implying increasing human, economic, and social costs (Patterson, 2018; Pickett et al., 2018). As a result of the disease-specific decrease in cognitive function and motor performance, dementia is a main reason for the loss of autonomy, and the accelerated need for help and institutionalization in aging (Bürge et al., 2017; World Health Organization \& Alzheimer's Disease International, 2012). Furthermore, the cognitive and physical deteriorations translate into hierarchical decline of activities of daily living (ADL) with progressing dementia (Johansen et al., 2020; Mlinac \& Feng, 2016). Instrumental ADL require higher cogni-

\section{Availability of data and material}

Due to data protection regulations of the Karlsruhe Institute of Technology, data and material will be only available from the corresponding author on reasonable request. tive demands (e.g., handling finances, going shopping) and are usually impaired at early dementia stages (Boyd, Wilks, \& Geiger, 2018; Martyr \& Clare, 2012). With the progression of the disease, additional problems with the performance of basic ADL (e.g., bathing, toileting, eating) arise (Boyd et al., 2018; Martyr \& Clare, 2012). These impairments lead to an elevated dependency in daily life, a reduction of quality of life (Giebel, Sutcliffe, \& Challis, 2015), and are related to a higher risk of falling in IWD (HenrySánchez, Kurichi, Xie, Pan, \& Stineman, 2012).

Between 50 and $80 \%$ of individuals in nursing homes are suffering from dementia, implying a higher care burden in terms of time and costs as compared to nursing home residents free of dementia. Studies found a relationship between deficits in ADL performance of individuals in nursing homes with perceived formal caregiver burden (Miyamoto, Tachimori, \& Ito, 2010; Sun, Mainland, Ornstein, Sin, \& Herrmann, 2018). Moreover, an increase of total resource use (hours per week) in nursing homes is associated with ADL dependence of nursing home residents (Sköldunger et al., 2019). Thus, there is a need for interventions which address improving or maintaining ADL performance in IWD in nursing homes.
High development costs and negative side effects of drug therapies in dementia care lead to an increased demand of nonpharmacological approaches like physical exercise (PE) interventions (Sopina \& Sørensen, 2018). The benefits of PE on cognition (e.g., executive function, memory), motor performance (e.g., strength, balance, gait performance), and ADL have been increasingly documented in the last few years (Forbes, Forbes, Blake, Thiessen, \& Forbes, 2015; Lam et al., 2018). Studies showed that PE interventions may improve or even slow down the progression of ADL deterioration in IWD living in nursing homes (Bossers et al., 2016; Bürge et al., 2017; Toots et al., 2016). Nevertheless, the results of the studies warrant caution of overinterpretation. Intervention periods ranging from 7 weeks up to 1 year, different settings (nursing homes, home care), different content or focus of PE interventions (only $\mathrm{PE}$ vs. combination of $\mathrm{PE}$ with cognitive training or social intervention), various training conditions (e.g., group vs. individuals training), and the implementation of different training parameters in terms of exercise duration and frequency, may complicate the comparison of study results (Forbes et al., 2015).

The primary aim of the present study was to investigate the effect of a 16-week multimodal exercise program (MEP) 
on ADL performance in IWD within a multicenter randomized controlled trial (RCT). To this end, we developed a dementia-specific MEP based on theoretical recommendations and a pilot study (Thurm et al., 2011). The intervention program combines both motor and cognitively stimulating exercises aiming at a higher efficacy on cognitive function, motor performance and ADL performance in IWD. Moreover, the MEP was developed for a sustainable implementation in nursing homes subsequent to the trial. We hypothesized that participants of the intervention group (IG) who underwent the MEP would change their ADL performance compared to participants of a control group (CG) who only received conventional treatment.

Considering the heterogeneous results of previous studies on the effects of $\mathrm{PE}$ interventions on ADL performance, our secondary aim was to explore the individual responses to our MEP with regard to ADL performance between positive-, negative-, and non-responders (positive-R, negative-R, and non-R). We hypothesized that responder groups would differ in terms of cognitive function and motor performance at baseline. In order to examine the complex interplay of ADL performance, we investigated motor and cognitive functions that may explain ADL performance. We hypothesized that various motor and cognitive assessments may explain the variability in ADL performance, and that these results may provide valuable implications for the design of further intervention studies.

\section{Methods}

This multicenter was developed and carried out by our research group at Karlsruhe in Germany. A 16-week MEP for individuals with mild to moderate dementia was implemented in 36 nursing homes in southwestern Germany. The MEP was developed based on a pilot study (Thurm et al., 2011) and a literature review (Scharpf, Servay, \& Woll, 2013). The intervention program combines both motor und cognitively stimulating exercises as well as the ritualiza- tion of the program sequences. Ethical approval for the study was provided by Karlsruhe Institute for Technology. The study was retrospectively registered in the German National Register of Clinical Trials (DRKS00010538). The following sections provide an abbreviated summary of the study methodology. A more detailed methodological study description, especially with regard to the MEP, can be found in the published study protocol (Trautwein, Scharpf, Barisch-Fritz, Niermann, \& Woll, 2017). The effects of the MEP on gait and motor performance have been published recently (Trautwein et al., 2020; Barisch-Fritz, Trautwein, Scharpf, Krell-Roesch, \& Woll, 2021), while the focus of this paper is on the effects on ADL performance.

\section{Study design and participants}

The multicenter randomized controlled trial included baseline and post-intervention assessments of cognition function, motor performance and ADL. Participants were allocated to the IG or CG using minimization software (MinimPy0.3 Saghaei \& Saghaei, 2011). A power analysis with $\mathrm{G}^{\star}$ Power 3 (Version 3.1.9.2, two-factor analysis of variance with repeated measurements, two groups, and two measurements, $\alpha=0.05, \beta=0.80$, $\left.\eta^{2}=0.01\right)$ resulted in a total sample size of 200 participants (Faul, Erdfelder, Lang, \& Buchner, 2007). We expected that $35 \%$ of participants would drop out of the study or have missing data. Thus, 270 participants for the total sample are required. As attendance and adherence to exercise interventions are not well documented in previous studies (Forbes et al., 2015), and a higher dropout rate was assumed in the IG (e.g., due to motivational problems), the sample of the IG was doubled. Hence, we aimed at a total sample size of 405 participants. Participants were identified by healthcare professionals in the nursing homes. Prior to baseline assessments, informed consent was signed by potential participants themselves or their legal guardian. Inclusion criteria were (1) diagnosis of primary dementia or "suspected dementia" (i.e., without a confirmed diagnosis or awaiting further clinical evaluation) verified by general practitioners and/or based on ICD-10 criteria, (2) Mini Mental State Examination (MMSE) with mild to moderate status (MMSE: 10-24), (3) age $>65$ years, (4) being able to walk for approximately 10 meters and (5) clearance by a general practitioner. Participants with secondary dementia, other severe cognitive impairments, neurological or severely acute diseases, severe motor impairments and/or no informed consent were excluded.

\section{Intervention}

The 16-week dementia-specific MEP was implemented, with two sessions per week, each lasting $60 \mathrm{~min}$. Every training session was designed as an imaginary journey with a ritualized arrival and departure element aiming to give participants orientation and familiarization. The mere exercise time included a combination of motor and cognitive tasks and took about $45 \mathrm{~min}$. In detail, strength, balance, endurance and flexibility tasks were performed at various durations and with medium to submaximal intensity. Small training devices, such as dumbbells (1-4.4 Ibs), sandbags ( 1 Ibs), skipping ropes or pool noodles where integrated into the exercises. Furthermore, different cognitive tasks were embedded in the MEP to stimulate cognitive functions, i.e., memory (e.g., remembering the destination of the last imaginary journey), attention (e.g., remembering a certain sequence of numbers), language (e.g., naming animals), and executive function (e.g., learning to perform according to visual or acoustic signals). Throughout the 16-week MEP, a progression of intensity of motor and cognitive exercises were planned and carried out, e.g., by increasing difficulty level or the number of exercise repetitions. Exercise intensity followed a predefined progression plan throughout the intervention period and was supervised by experienced instructors. Examples of different motor and cognitive tasks and their progression are presented in the appendix (Supplementary Table 1). In order to achieve a high degree of standardization, all instructors received a detailed manual of the MEP and underwent a special training pro- 
gram concerning the content of the MEP as well as the characteristics of the study sample. Furthermore, only instructors with theoretical and practical knowledge in sport science guided the MEP during the intervention study.

\section{Outcomes}

ADL (primary outcome measure) were assessed by a proxy-based questionnaire, the Barthel Index (BI; Mahoney \& Barthel, 1965) and two performancebased assessments, i.e., the Erlangen Test of Activities of Daily Living (E-ADL; Graessel et al., 2009) and the 7-item Physical Performance Test (PPT-7; Reuben \& Siu, 1990). The BI is a widely used questionnaire in geriatric care to assess functioning of older individuals in daily life. Usually, it is completed by caregivers and contains ten items: feeding, moving from a wheelchair to bed and return, personal toilet, getting on and off toilet, bathing, walking on level surface, ascend and descend stairs, dressing, controlling bowels and controlling bladder. The BI sum score ranges from 0 (totally dependent) to 100 (independent) (Mahoney \& Barthel, 1965). The PPT-7 contains seven activities of everyday life: 50 -feet walk, putting on and removing a jacket, simulated eating, writing a sentence, putting a book into a shelf, a $360^{\circ}$ turn, and picking up a penny from the floor. The maximum attainable score of the PPT-7 is 28 , with a higher score indicating a better ADL performance (Reuben \& Siu, 1990). The PPT-7 was found to be feasible and reliable in IWD (Farrell, Rutt, Lusardi, \& Williams, 2010). The $\mathrm{E}-\mathrm{ADL}$ is the only ADL assessment that was specifically developed for IWD. It is performance-based and comprises five typical routines of daily life: cutting bread, pouring a drink, opening a cupboard, washing hands and tying a bow. The total score of the E-ADL ranges from 0-30, with a higher score representing better results. The E-ADL has good validity and test-retest reliability in IWD (Graessel et al., 2009).

For the explorative analysis of responder groups and underlying functions of ADL performance, we defined cognitive function and motor performance as sec-

Ger J Exerc Sport Res 2021 · 51:506-517 https://doi.org/10.1007/s12662-021-00760-4

(c) The Author(s) 2021

\section{J. Bezold · S. Trautwein · B. Barisch-Fritz · A. Scharpf · J. Krell-Roesch · C. R. Nigg · A. Woll \\ Effects of a 16-week multimodal exercise program on activities of daily living in institutionalized individuals with dementia. A multicenter randomized controlled trial}

\section{Abstract}

We aimed to examine the effects of a 16week multimodal exercise program (MEP) on activities of daily living (ADL) in individuals with dementia (IWD). Furthermore, we investigated the participants' individual response to the MEP and whether baseline cognitive and motor performance explain ADL performance. We conducted a multicenter randomized controlled trial (RCT) involving 319 participants aged $\geq 65$ years with mild to moderate dementia. ADL were assessed at baseline and after the 16-week intervention using the Barthel Index $(\mathrm{BI})$, the Erlangen Test of Activities of Daily Living (E-ADL) and the 7-item Physical Performance Test (PPT7). We additionally assessed cognitive and motor performance using standardized and validated assessments. Intervention effects were examined through two-factor analysis of variance with repeated measurements applying a per protocol and an intention-to-treat analysis. We compared baseline cognitive and motor performance between positiveresponders (positive-R), non-responders (non- $R$ ), and negative-responders (negative$R$ ) and examined cognitive and motor performance as potential cofounders of ADL by conducting multiple regression analyses. There were no significant timexgroup effects on ADL. Between 20 and $32 \%$ of participants responded positively to the intervention, i.e., improved ADL performance from baseline to follow-up. Positive-R had worse baseline motor performance compared to non- $R$. Cognitive and motor performance explained up to $51.4 \%$ of variance in ADL. The MEP had no significant overall effect on ADL in IWD. This may be related to insufficient exercise intensity. However, our results indicate that the response to the MEP depends on individual prerequisites which should thus be considered in further research on individual exercise approaches.

\section{Keywords}

Older adults · Cognitive impairment · Physical exercise $\cdot$ Nursing home $\cdot$ Intervention study ondary outcomes. We assessed overall cognition with the Mini Mental State Examination (MMSE; Folstein, Folstein, \& McHugh, 1975), learning and memory with Digit Span forward and backward (Wilde, Strauss, \& Tulsky, 2004), processing speed with the Trail Making Test Part A (TMT-A; Reitan, 1958), and visual spatial function with the Clock Drawing Test (CDT; Shulman, Shedletsky, \& Silver, 1986). To assess motor performance, we used the modified 30-second chair stand test (30s CST) for lower limb strength (Blankevoort, van Heuvelen, \& Scherder, 2013), the Short Physical Performance Battery (SPPB; Guralnik et al., 1994) for lower limb function, Frailty and Injuries: Cooperative Studies of Intervention Techniques (FICSIT-4) for balance (Rossiter-Fornoff, Wolf, Wolfson, \& Buchner, 1995), and Timed-Up and Go (TUG; Podsiadlo \& Richardson, 1991) as well as a 6-meter walk test (6MWT; Graham, Ostir, Kuo, Fisher, \& Ottenbacher,
2008) for mobility. The reader is referred to the study protocol (Trautwein et al., 2017) for a more detailed description of all motor and cognitive assessments. Feasibility of the motor assessments were discussed with an international expert panel before the conduct of this study. Furthermore, a standardized testing procedure was developed by the expert panel (Trautwein et al., 2019). Subsequently, the applied test battery has been checked for feasibility in IWD.

\section{Statistical analysis}

Statistical analysis was done with SPSS 27.0 (IBM Corporation, Armonk, NY, USA) for Windows with alpha ( $\alpha$ ) set a priori at 0.05 for all tests. To investigate the effect of the MEP on $\mathrm{ADL}$ performance between IG and CG, we using two different approaches (per protocol, intention to treat). This approach was also addressed but not further speci- 
fied within the study protocol (Trautwein et al., 2017). First, a per protocol analysis was performed including all participants with sufficient attendance to the MEP (only IG: $\geq 75 \%$, at least 24 of 32 trainings sessions) and a complete assessment (IG and CG: baseline, post) of at least one ADL assessment. In IWD, attendance to PE interventions and possible barriers (e.g., illness, previous exercise experience) vary widely (van der Wardt et al., 2017). A minimum of $75 \%$ for the per protocol analysis was defined on the basis of mean attendance rates of previously published studies with similar intervention period, duration, and frequency of training sessions, as well as sample sizes (Telenius, Engedal, \& Bergland, 2013; Toots et al., 2016). Second, we imputed missing data of the primary outcomes for an intention-to-treat analysis using multiple imputation (fully conditional specification imputation method, ten imputations, ten iterations). Data of deceased participants was not imputed. ADL performance was considered as primary outcome and motor performance and cognitive function were considered as predictors. Furthermore, to ensure plausibility of data, other constraints like minimum and maximum values in each variable according to observed ranges, rounding according to original data, 100 maximal case draws and ten maximal parameter draws were defined. We considered pooled results as provided by SPSS. If SPSS did not support this pooling procedure for several statistical analyses, we reported ranges observed throughout the imputations.

Before carrying out statistical analyses, required assumptions were tested. Differences in baseline characteristics between IG and CG were calculated using unpaired T-tests, Chi-square $\left(X^{2}\right)$ tests and Mann-Whitney $U$ tests. Normally distributed data are presented as means (M) and standard deviations (SD). To investigate group-, time- and interaction effects of the MEP, twoway repeated measures analysis of variance (ANOVA) and paired T-tests were calculated for primary outcomes. The calculated effect sizes are Cohen's $d$ for paired and unpaired T-tests and $\eta_{\mathrm{p}}{ }^{2}$ for two-way repeated measures ANOVA.
Furthermore, we applied an explorative approach for the secondary aim, i.e., the responder-analysis. Previous studies often focused on main effects and group differences; however, the need to consider individual responses to exercise programs in IWD is currently discussed (Yu, Salisbury, \& Mathiason, 2021). We decided against a simple "responder" and "non-responder" division as proposed in a previously published article (Müllers, Taubert, \& Müller, 2019), and additionally defined a group with maintaining ADL performance, which may be considered as partial success of physical exercise in IWD. To this end, we divided the IG of the per protocol sample into three groups based on distribution-based methods (i.e., information about the standard measurement error of the assessments within the per protocol analysis): positive- $\mathrm{R}$ (positive change of $10 \%$ and more in ADL performance), negative- $\mathrm{R}$ (negative change of $10 \%$ and more in ADL performance), and non- $\mathrm{R}$ (change between positive and negative $10 \%$ in ADL performance). We compared baseline cognitive function and motor performance between these responder groups using Kruskall-Wallis test, and one-factor ANOVA. Dunn-Bonferroni tests and Tukey-Kramer post hoc tests were used for post hoc analysis. For the analysis of underlying mechanisms of ADL performance, multiple regression analysis with stepwise selection were used with BI, E-ADL, and PPT-7 as dependent variables, and the already mentioned motor and cognitive assessments as independent variables. The calculated effect size was $\mathrm{f}^{2}$.

\section{Results}

\section{Sample}

In all, 600 IWD were screened for eligibility of study participation between March 2015 and March 2017, and 319 persons were considered as suitable for the study: 201 participants were randomly allocated to the IG and 118 participants to the CG after baseline assessment. Please refer to - Fig. 1 for a flow chart of participants. Dropout rate in both IG and CG was 8\%.
Baseline characteristics of the per protocol sample

- Table 1 presents the baseline characteristics of the per protocol sample $(n=191)$. The mean age of the participants was 86 years (SD 6 years) and $86 \%$ of the sample was female. A mean MMSE value of 17 (SD 4) indicated moderate cognitive impairment at baseline. More than three quarters of the per protocol sample used walking aids (walker: 70\%; walking sticks: $8 \%$ ). Mean value on the CIRS morbidity index was 9 (SD 5) and the mean number of required medications was 7 (SD 4). In $22 \%$ of the per protocol sample, the intake of antidementia medication was confirmed. Statistically significant differences in baseline characteristics of the per protocol sample between IG and CG were observed for the use of walking aids $\left(X^{2}(2)=6.254, p=0.044\right)$, number of medications $(\mathrm{t}(148)=2.622, p=0.010)$, and body mass index $(\mathrm{t}(178)=2.041$, $p=0.043)$. Baseline characteristics of the intention-to-treat sample are provided in the Appendix (Supplementary Table 2).

\section{Effects of the MEP on ADL performance}

\section{Per protocol sample}

Participants of the IG in the per protocol sample had a mean adherence of $91 \%$. Mean values and SD of primary outcomes are presented in - Table 2. At baseline, no statistically significant differences between IG and CG in either ADL assessments (Barthel Index, E-ADL, PPT-7) were observed ( $p>0.05$ for all). We did not observe any statistically significant time $\times$ group effects ( $p>0.05, \eta_{\mathrm{p}}{ }^{2}=0.004-0.019 ;$ - Table 3$)$. Also, there were no significant withingroup time effects from baseline $\left(\mathrm{t}_{0}\right)$ to post-measurement $\left(t_{1}\right)$ in either ADL assessments for the IG.

\section{Intention-to-treat analysis}

An analysis of missing data indicated missing values ranging between $3.7 \%$ (PPT-7 at baseline) and $23.6 \%$ (BI at baseline), and 226 of 319 data sets were incomplete with respect to ADL performance. Two-way repeated measures 


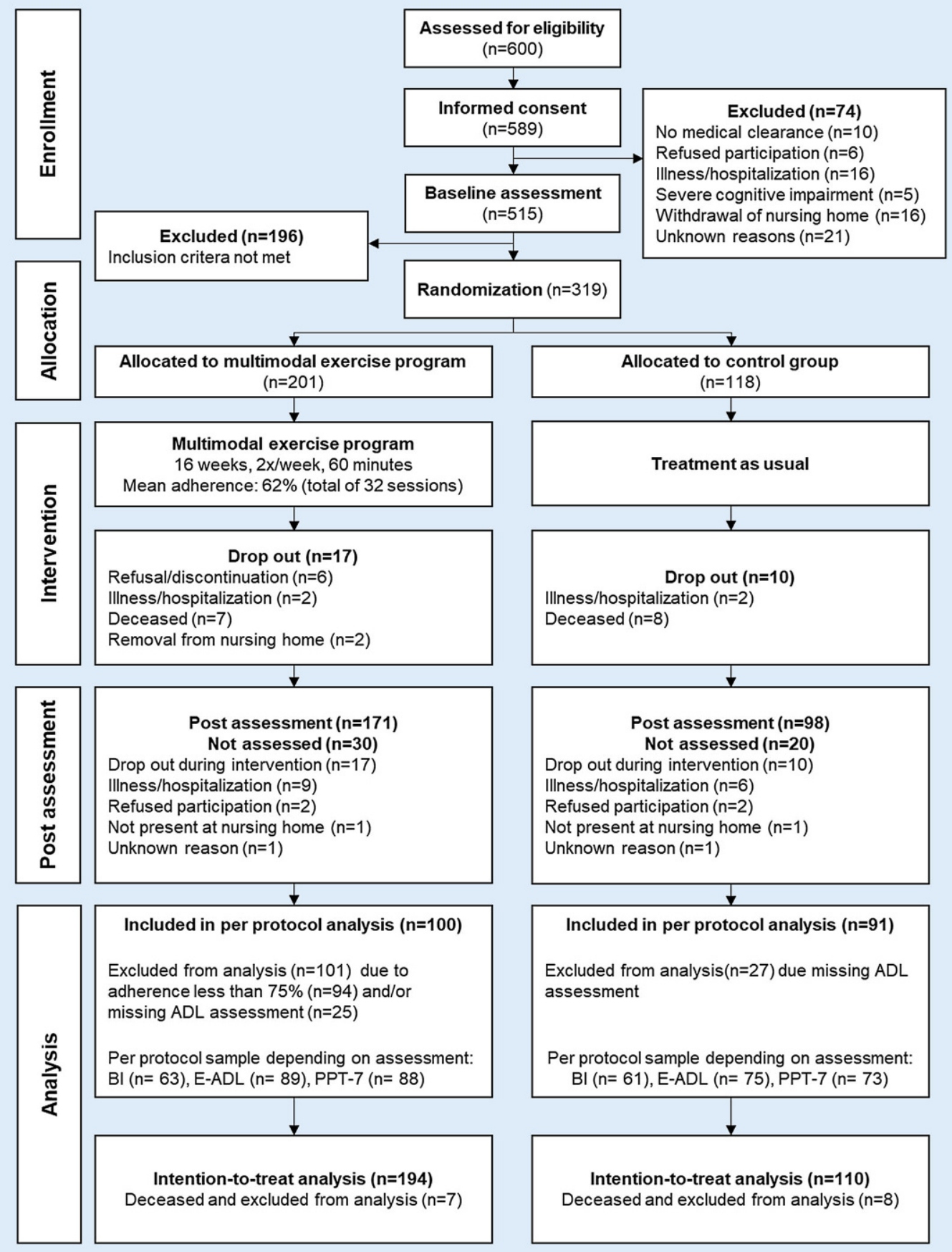

Fig. $1<$ Flow of participants. ADL Activities of Daily Living, $B /$ Barthel-Index, E-ADL Erlangen Test of Activities of Daily Living, $n$ number, $P$ PT-7 7-item Physical Performance Test

ANOVAs showed no statistically significant timexgroup effects for either ADL outcome ( $\left.p>0.05, \eta p^{2}=0.000-0.013\right)$. For E-ADL, the intervention group showed a statistically significant decrease from baseline to post assessment $(\mathrm{t}(277)=2.301, p=0.022)$. We observed no other statistically significant withingroup time effects. Details of the statistical analysis of the intention-to-treat sample are shown in the Appendix (Supplementary Table 3).

\section{Explorative responder-analysis (per protocol, intervention group)}

A responder analysis in the intervention group of the per protocol sample showed that $20.2 \%$ (E-ADL), 30.7\% (PPT-7), and $31.7 \%$ (BI) of participants of the IG responded positively to the
MEP (Supplementary Table 4). The majority of participants of the IG showed no changes in the primary outcomes (50.0-68.3\%), and between 19.3 and $27.0 \%$ had a decrease in their ADL performance that was greater than $10 \%$. With regard to the Barthel Index, no negative-responders were observed (Supplementary Table 4). The comparison of baseline values in cognitive function and motor performance between differ- 


\begin{tabular}{|c|c|c|c|c|}
\hline & $\begin{array}{l}\text { Total sample } \\
{[n=191]}\end{array}$ & $\begin{array}{l}\text { IG } \\
{[n=100]}\end{array}$ & $\begin{array}{l}\text { CG } \\
{[n=91]}\end{array}$ & $\begin{array}{l}\text { Group differences } \\
{\left[t(d f) / z / X^{2}(d f), p\right]}\end{array}$ \\
\hline $\begin{array}{l}\text { Age, years } \\
{[\mathrm{M}(\mathrm{SD}), \text { range] }}\end{array}$ & 86 (6) 67-98 & 85 (7) 67-97 & 87 (5) 70-98 & $\mathrm{t}(189)=1.886, p=0.061$ \\
\hline Sex, female & $86 \%$ & $83 \%$ & $89 \%$ & $X^{2}(1)=1,418, p=0.234$ \\
\hline \multicolumn{5}{|l|}{ Diagnosis of dementia } \\
\hline Yes & $70 \%$ & $71 \%$ & $68 \%$ & \multirow[t]{3}{*}{$X^{2}(2)=2.701, p=0.259$} \\
\hline No & $17 \%$ & $19 \%$ & $14 \%$ & \\
\hline Unknown & $13 \%$ & $10 \%$ & $18 \%$ & \\
\hline \multicolumn{5}{|l|}{ Type of dementia } \\
\hline Alzheimer's disease & $19 \%$ & $18 \%$ & $19 \%$ & \multirow[t]{5}{*}{$X^{2}(4)=7.948, p=0.094$} \\
\hline Vascular dementia & $23 \%$ & $31 \%$ & $15 \%$ & \\
\hline Mixed dementia & $4 \%$ & $3 \%$ & $6 \%$ & \\
\hline Other & $2 \%$ & $3 \%$ & $0 \%$ & \\
\hline No/unknown diagnosis & $52 \%$ & $45 \%$ & $60 \%$ & \\
\hline MMSE [M (SD), range] & $17(4), 10-24$ & $17(4), 10-24$ & $17(4), 10-24$ & $\mathrm{t}(188.103)=0.619, p=0.537$ \\
\hline \multicolumn{5}{|l|}{ Use of walking aid } \\
\hline Walker & $70 \%$ & $63 \%$ & $77 \%$ & \multirow[t]{3}{*}{$X^{2}(4)=7.948, p=0.094$} \\
\hline Waking stick(s) & $8 \%$ & $8 \%$ & $9 \%$ & \\
\hline No walking aid & $22 \%$ & $29 \%$ & $14 \%$ & \\
\hline \multicolumn{5}{|l|}{ CIRS [M (SD), range] } \\
\hline Morbidity Index & $9(5), 1-26$ & $9(4), 1-20$ & $10(6), 2-26$ & $\mathrm{t}(113)=0.798, p=0.426$ \\
\hline Severity Index & $1.6(0.4), 1-3$ & $\begin{array}{l}1.5(0.4), 1-3 \\
\text { Not available for 33\% }\end{array}$ & $\begin{array}{l}1.6(0.5), 1-3 \\
\text { Not available for } 47 \%\end{array}$ & $z=-0.202, p=0.840$ \\
\hline $\begin{array}{l}\text { Number of medications } \\
\text { [M (SD), range] }\end{array}$ & $7(4), 0-27$ & $8(4), 1-27$ & $6(4), 0-20$ & $t(148)=2.622, p=0.010$ \\
\hline $\begin{array}{l}B M I, \mathrm{~kg} / \mathrm{m}^{2} \\
{[\mathrm{M}(\mathrm{SD}), \text { range }]}\end{array}$ & $\begin{array}{l}27.8(4.6) \\
17.6-48.5\end{array}$ & $28.5(4.4), 19.7-48.5$ & $27.1(4.8), 17.6-38.0$ & $t(178)=2.041, p=0.043$ \\
\hline
\end{tabular}

Table 2 Mean values and standard deviations of primary outcomes

\begin{tabular}{llllllllll} 
& & \multicolumn{3}{c}{ Baseline } & \multicolumn{3}{c}{ Post } & \multicolumn{3}{c}{ Difference baseline-post } \\
& & Mean & SD & Mean & SD & Mean & SD & Cl95 \\
\hline BI & IG $(n=63)$ & 69.7 & 18.7 & 72.3 & 18.8 & -2.619 & 14.420 & $-6.251-1.013$ \\
& CG $(n=61)$ & 67.2 & 20.2 & 65.6 & 21.5 & 1.639 & 16.475 & $-2.580-5.859$ \\
E-ADL & IG $(n=89)$ & 26.3 & 4.3 & 25.9 & 4.3 & 0.416 & 4.293 & $-0.489-1.320$ \\
& CG $(n=75)$ & 26.6 & 4.1 & 26.7 & 3.5 & -0.107 & 4.444 & $-1.129-0.916$ \\
PPT-7 & IG $(n=88)$ & 12.7 & 4.7 & 13.0 & 5.6 & -0.318 & 3.647 & $-1.091-0.455$ \\
& CG $(n=73)$ & 12.4 & 4.4 & 11.8 & 4.7 & 0.548 & 3.606 & $-0.293-1.389$
\end{tabular}

BI Barthel Index, CG control group, E-ADL Erlangen Test of Activities of Daily Living, IG intervention group, $n$ number, PPT-7 7-item Physical Performance Test, $S D$ standard deviation

ent groups of $\mathrm{ADL}$ responders revealed statistically significant results for $\mathrm{BI}$ and E-ADL performance (- Table 4). With regard to the Barthel Index, positive-R performance at baseline was significantly worse compared to non- $\mathrm{R}$ for SPPB $(p=0.002, \quad d=-0.993), \quad 30-S T S$ $(p=0.008, \mathrm{~d}=-0.786)$ and FICSIT-4 $(p=0.036, \quad \mathrm{r}=-0.378) . \quad$ A one-way
ANOVA revealed significant differences between E-ADL responder groups for FICSIT-4, and CDT. The post hoc analysis showed statistically significantly worse performance of positive-responders in FICSIT-4 at baseline, compared to nonresponders $\left(p=0.012, \mathrm{MD}=-1.146, \mathrm{CI}_{95}\right.$ [ -2.084 to 0.208$])$. For CDT, the post hoc analysis was not significant. We found no statistically significant differences between PPT-7 responders for baseline motor and cognitive performance.

\section{Explorative regression analysis (per protocol sample)}

For baseline Barthel Index, weak to moderate correlations with baseline cognitive function and motor performance were found $(|\mathrm{r}|=0.141-0.349, p<0.05)$. In a multiple regression analysis model, baseline FICSIT-4 and TUG were statistically significant regression coefficients $(p<0.05)$. This model explained $14.3 \%$ of variance in baseline $\mathrm{BI}(\mathrm{F}(2$, $152)=13.819, p<0.005)$. For baseline E-ADL, weak correlations with baseline cognitive function and motor performance were found $(|\mathrm{r}|=0.118-0.170$, $p<0.05)$ and only baseline MMSE was a statistically significant coefficient in 
Table 3 Effects of the MEP on ADL performance (per protocol sample)

\begin{tabular}{|c|c|c|c|c|c|c|c|c|c|}
\hline & & \multicolumn{2}{|c|}{ Baseline differences } & \multicolumn{3}{|c|}{ Within-group effects } & \multicolumn{3}{|l|}{ Timexgroup effects } \\
\hline & & $\mathbf{t}(\mathbf{d f})$ & $p$ & $\mathbf{t}(\mathbf{d f})$ & $p$ & Effect size $d$ & $\begin{array}{l}F \\
\left({ }^{\prime} f_{\text {numerator, }}\right. \\
\left.d_{\text {denominator }}\right)\end{array}$ & $p$ & Effect size $\eta_{p}{ }^{2}$ \\
\hline \multirow[t]{2}{*}{$\mathrm{BI}$} & IG $n=63$ & \multirow[t]{2}{*}{$t(122)=0.707$} & \multirow[t]{2}{*}{0.481} & $t(62)=-1.442$ & 0.154 & 0.254 & \multirow[t]{2}{*}{$F(1,122)=2.350$} & \multirow[t]{2}{*}{0.128} & \multirow[t]{2}{*}{0.019} \\
\hline & CG $n=61$ & & & $t(60)=0.777$ & 0.440 & 0.015 & & & \\
\hline \multirow[t]{2}{*}{ E-ADL } & IG $n=89$ & \multirow[t]{2}{*}{$t(162)=0.445$} & \multirow[t]{2}{*}{0.657} & $t(88)=0.914$ & 0.363 & 0.106 & \multirow[t]{2}{*}{$F(1,162)=0.584$} & \multirow[t]{2}{*}{0.446} & \multirow[t]{2}{*}{0.004} \\
\hline & CG $n=75$ & & & $t(74)=-0.208$ & 0.836 & 0.052 & & & \\
\hline \multirow[t]{2}{*}{ PPT-7 } & IG $n=88$ & \multirow[t]{2}{*}{$t(159)=0.469$} & \multirow[t]{2}{*}{0.640} & $t(87)=-0.818$ & 0.415 & 0.087 & \multirow[t]{2}{*}{$F(1,159)=2.274$} & \multirow[t]{2}{*}{0.134} & \multirow[t]{2}{*}{0.014} \\
\hline & CG $n=73$ & & & $t(72)=1.298$ & 0.198 & 0.152 & & & \\
\hline
\end{tabular}

BI Barthel Index, CG control group, CI95 95\% confidence interval, $d f$ degrees of freedom, E-ADL Erlangen Test of Activities of Daily Living, IG intervention group, $M$ mean, $n$ number, PPT-7 7-item Physical Performance Test, SD standard deviation, MEP multimodal exercise program

\begin{tabular}{|c|c|c|c|c|c|}
\hline & \multirow{2}{*}{$\begin{array}{l}\text { Positive-R } \\
\text { Mean (SD) }\end{array}$} & \multirow{2}{*}{$\begin{array}{l}\text { Non-R } \\
\text { Mean (SD) }\end{array}$} & \multirow{2}{*}{$\begin{array}{l}\text { Negative-R } \\
\text { Mean (SD) }\end{array}$} & \multirow{2}{*}{$\begin{array}{l}\text { Between group difference } \\
F\left(d f_{\text {numerator, }} \mathbf{d f}_{\text {denominator }}\right) / \mathbf{X}^{2}(\mathbf{d f}) / \mathbf{t}(\mathbf{d f}) / \mathbf{z}, p\end{array}$} & \multirow[t]{2}{*}{ Post hoc analysis } \\
\hline & & & & & \\
\hline \multicolumn{6}{|l|}{$B I$} \\
\hline $\operatorname{SPPB}(n=48)$ & $5.4(3.1)$ & $7.8(2.3)$ & - & $\mathrm{t}(46)=-3.243, p=0.002, \mathrm{~d}=-0.993$ & - \\
\hline 30 s CST $(n=52)$ & $7.1(3.1)$ & $9.7(3.3)$ & - & $\mathrm{t}(50)=-2.758, p=0.008, \mathrm{~d}=-0.786$ & - \\
\hline FICSIT $(n=59)$ & $1.7(1.3)$ & $2.6(1.5)$ & - & $z=-2.907, p=0.036, r=-0.378$ & - \\
\hline \multicolumn{6}{|l|}{$E-A D L$} \\
\hline FICSIT $(n=88)$ & $1.5(1.3)$ & $2.7(1.4)$ & $2.1(1.4)$ & $\mathrm{F}(2,85)=4.474, p=0.014, n p^{2}=0.095$ & $\begin{array}{l}p=0.012, M D=-1.146 \\
C l_{95}[-2.084-0.208]^{a}\end{array}$ \\
\hline $\mathrm{CDT}(n=82)$ & $2.5(1.0)$ & $3.3(1.3)$ & $2.7(1.4)$ & $X^{2}(2)=6.070, p=0.048$ & n.s. \\
\hline
\end{tabular}

multiple regression analysis $(p<0.05)$. The model explained $2.4 \%$ of the variance in baseline E-ADL performance $(\mathrm{F}(1,197)=5.843, \quad p=0.017) . \quad$ For baseline PPT-7, weak to high correlations with baseline cognitive function and motor performance were found $(|\mathrm{r}|=0.198-0.609, p<0.05)$. In multiple regression analysis, a model with TUG, gait speed calculated from the 6MWT, FICSIT-4, 30-STS and DS forward as coefficients explained $51.4 \%$ of the variance in PPT-7 performance $(\mathrm{F}(5$, 196) $=43.581, p<0.005)$. Please refer to - Table 5 for the results of the multiple regression analyses.

\section{Discussion}

\section{Effects of the MEP on ADL performance}

The primary aim of this paper was to investigate the influence of a 16-week multimodal exercise program on ADL performance in IWD. We did not ob- serve any significant time-group effects $\left(\eta_{\mathrm{p}}{ }^{2}: 0.004-0.019\right)$ of the 16-week MEP on ADL performance in IWD. Therefore, we could not confirm our hypothesis that participants of the MEP improved their ADL performance compared to the CG. Previous studies that investigated the effects of PE on ADL performance in IWD living in nursing homes yielded heterogeneous results. While some studies did not find any significant effects (Henskens, Nauta, Drost, \& Scherder, 2018; Lamb et al., 2018), others found a slowed deterioration of ADL compared to a control group (Bürge et al., 2017; Littbrand, Lundin-Olsson, Gustafson, \& Rosendahl, 2009; Rolland et al., 2007; Toots et al., 2016) or positive effects of PE on ADL performance (Bossers et al., 2016). Nevertheless, all these studies differed in terms of sample characteristics, setting, intervention period and intervention content, hampering a comparison and a critical handling with these findings is thus recommended (Forbes et al., 2015). Studies that yielded posi- tive effects had longer intervention periods, higher training frequency, smaller groups or one-to-one sessions, and/or an adaption of intensity of exercise content during the intervention. We could not reach our aim to increase intensity throughout the intervention, which may be a main reason for our non-significant results. Moreover, even though two training sessions are recommended for nursing home residents (de Souto Barreto et al., 2016), this was feasible for our sample but might not have been sufficient. Furthermore, the baseline differences in walking-aid use were not further addressed within our analysis, but may have influenced the intensity adaption throughout our intervention period. Instructing a group in which some IWD use walking aids and others do not may have complicated the implementation of intensity adaptions, especially with regard to ensuring the safety of the participants. These mentioned challenges underline the difficulty to adapt exercise intensities in a highly heterogeneous sample. 


\begin{tabular}{|c|c|c|c|c|c|c|c|c|c|}
\hline & \multicolumn{5}{|c|}{ Coefficients } & \multicolumn{4}{|l|}{ Model } \\
\hline & B & SE & $\boldsymbol{\beta}$ & $\mathbf{t}$ & $p$ & $\mathbf{R}^{2}$ & Adjusted $\mathbf{R}^{2}$ & $F\left(d f_{\text {numerator, }}, \mathrm{df}_{\text {denominator }}\right), p$ & $\mathbf{f}^{2}$ \\
\hline \multicolumn{10}{|l|}{$B I(n=155)$} \\
\hline Constant & 70.985 & 4.896 & - & 14.497 & $<0.001$ & - & - & - & - \\
\hline FICSIT & 3.801 & 1.143 & 0.271 & 3.326 & 0.001 & - & - & - & - \\
\hline TUG & -0.348 & 0.145 & -0.196 & -2.406 & 0.017 & - & - & - & - \\
\hline Model & & & & & & 0.154 & 0.143 & $F(2)=13.819, p<0.001$ & 0.182 \\
\hline \multicolumn{10}{|l|}{$E-A D L(n=199)$} \\
\hline Constant & 24.689 & 1.071 & - & 23.044 & $<0.001$ & - & - & - & - \\
\hline MMSE & 0.145 & 0.060 & 0.170 & 2.417 & 0.017 & - & - & - & - \\
\hline Model & & & & & & 0.029 & 0.024 & $\mathrm{~F}(1)=5.843, p=0.017$ & 0.029 \\
\hline \multicolumn{10}{|l|}{ PPT-7 $(n=202)$} \\
\hline Constant & 5.330 & 1.822 & - & 2.926 & 0.004 & - & - & - & - \\
\hline TUG & -0.095 & 0.029 & -0.236 & -3.310 & 0.001 & - & - & - & - \\
\hline Walking Speed & 6.563 & 1.517 & 0.291 & 4.328 & $<0.001$ & - & - & - & - \\
\hline FICSIT & 0.703 & 0.174 & 0.216 & 4.033 & $<0.001$ & - & - & - & - \\
\hline 30s CST & 0.204 & 0.070 & 0.170 & 2.894 & 0.004 & - & - & - & - \\
\hline DS, counting forward & 0.491 & 0.184 & 0.132 & 2.676 & 0.008 & - & - & - & - \\
\hline Model & & & & & & 0.526 & 0.514 & $F(5)=43.581, p<0.001$ & 1.120 \\
\hline
\end{tabular}

Another reason for our non-significant results may be the high variability of ADL performance that was also reported in a previous study (Bürge et al., 2017). Moreover, even though the BI, E-ADL and PPT-7 were used in other studies with IWD before, it must be noted that they may not be specific enough to detect subtle changes in ADL performance in IWD. The sensitivity of the BI and its objectivity in assessing ADL performance of IWD living in nursing homes must be seen critical (Yi et al., 2020). Nevertheless, we decided to use the BI as a proxy-based evaluation tool, and to include an external assessment in addition to the performance-based tests as they may have been influenced by daily form or mood of the participants. However, we are aware that the BI may not have captured the effects of our MEP. Moreover, the E-ADL was rated as too easy for individuals with mild dementia (Luttenberger et al., 2012). Originally, the PPT-7 was not developed for IWD and therefore may not be feasible to detect small changes from baseline to post measurement as in our study. Nevertheless, previous longitudinal studies showed that ADL performance of IWD residing in long-term care facilities deteriorates over time (Johansen et al., 2020). Therefore, a small improvement or even maintenance of ADL performance over time could be a valuable outcome.

Indeed, our explorative responderanalysis revealed between 20.2 and $31.7 \%$ positive-responders with regard to $\mathrm{ADL}$ performance in the IG. Nevertheless, the majority of the participants did not respond to the MEP (i.e., non-R, between 50 and 68\%). We observed worse motor performance (balance, mobility, and lower limb function) in positive- $R$ compared to non-R. These results imply that the intensity of our MEP worked well for individuals with weak baseline performance. From a training science point of view, it is crucial to individually modify intensity of interventions in order to achieve an improvement (Bürge et al., 2017; Littbrand et al., 2009; Littbrand, Stenvall, \& Rosendahl, 2011). Originally, our MEP was designed to be carried out with increases of intensity of $\mathrm{PE}$ to achieve adaptions in participants of the IG. Due to the high heterogeneity of our sample in terms of disease severity, age, and other personal characteristics, we had to intensify our safety efforts and the majority of exercises were carried out while participants were seated. This resulted in lower training stimuli and may be one explanation as to why less performance adaptions might have been achieved. This consideration is supported by the fact that previous research found positive effects of $\mathrm{PE}$ on $\mathrm{ADL}$ performance when $\mathrm{PE}$ was provided in one-to-one guided sessions (Bossers et al., 2016). Therefore, more personcentered approaches considering individual skills and impairments may be warranted (Prizer \& Zimmerman, 2018) and an individualization of instructions depending on the degree of cognitive impairment may be also useful. These factors underline the need for individualized MEPs tailored to the specific needs of an IWD in order to impact physical performance and cognitive function. Indeed, the concept of individualized medicine which has already become popular in prevention and treatment of Alzheimer's disease (Hampel et al., 2017) is also transferable to the design and conduct of individualized exercise programs for IWD (Müllers et al., 2019). Of note, the feasibility of individualized exercise programs for IWD in nursing homes 
has to be discussed, as time available for $\mathrm{PE}$ programs in geriatric care settings is often limited. Organizational and structural aspects from both nursing homes and health care systems could support and facilitate the implementation of $\mathrm{PE}$ interventions in nursing homes (e.g., by engaging volunteers) (de Souto Barreto et al., 2016). While previous studies have shown the cost-effectiveness of PE interventions for IWD in community settings (Nickel, Barth, \& KolominskyRabas, 2018), the evaluation of costs and personal resources in nursing homes is lacking to date. One may only speculate that individualized PE interventions may require more personal resources at first, but save financial and personal resources in the long-term if they are effective. Mobile health applications may therefore be an effective and efficient possible solution in the implementation of individualized exercise programs in IWD (Barisch-Fritz et al., 2020). In detail, they may represent an easy to administer tool that could support nursing home staff, while conducting PE interventions with information on exercises, training plans, possible risk factors or required training material. Furthermore, mobile health applications offer the opportunity to individually adapt PE programs for example by integrating data-based or artificial intelligence algorithms (Helbostad et al., 2017). Of note, the monitoring of exercise intensity may be a further advantage of mobile health applications. Previous research used and recommended a combination of heart-rate monitoring and rating of perceived exertion (Sanders et al., 2020), but this was mainly done by research assistants and may be too time-consuming for nursing home staff. For a feasible and applicable monitoring of exercise intensity in IWD, a protocol of exercise repetitions or exercise time, as well as externally rated exertion by nursing home staff could be implemented in a mobile health application. Despite all these advantages and possibilities of mobile health interventions, studies examining their usability and feasibility of in nursing homes are sparse (BarischFritz et al., 2020).
Our MEP was originally designed to primarily improve cognitive function and motor performance (Barisch-Fritz et al., 2021; Trautwein et al., 2020), and to address ADL performance indirectly. PE interventions which include ADL-specific tasks and take into account the complex requirements of $\mathrm{ADL}$ performance could be more effective. Therefore, we investigated the underlying mechanisms of ADL performance using multiple regression analysis. Explained variance ranged from $2.4 \%$ (EADL) to $51.4 \%$ (PPT-7) with statistically significant coefficients for both cognitive function and motor performance. The small explanation of variance in E-ADL was expected, as the test contains tasks for upper extremities that were not captured with our assessment battery (Graessel et al., 2009). Nevertheless, our results may contribute to a deeper understanding of different sub-dimensions of ADL performance. If relevant cognitive and motor functions are carefully selected for the conceptualization of PE interventions, potential transfer effects on ADL performance could be more beneficial (Hagovska \& Nagyova, 2017). Our results showed that assessments for balance and mobility explained the variance in the BI. For the performance of the PPT-7, walking speed, lower limb strength, and memory were additionally important. These results are partly in line with previous studies (GarciaPinillos, Cozar-Barba, Munoz-Jimenez, Soto-Hermoso, \& Latorre-Roman, 2016; Portegijs, Rantakokko, Viljanen, Sipilä, \& Rantanen, 2016). However, beyond cognitive function and motor performance, other factors like having depression or feeling pain during specific tasks of daily life (e.g., sit-to-stand transfers) may influence ADL performance in IWD (Mlinac \& Feng, 2016) and should be considered in the conceptualization of PE interventions.

\section{Strengths and limitations}

The strengths of our study are the high methodological quality and the large sample size. Furthermore, we implemented a MEP that was based on theoretical considerations and proved to be feasible conducted in a sample of IWD living in nursing homes within a pilot study. In addition, we designed the MEP to be highly suitable in everyday life of nursing homes. Our previously assumed dropout rate was lower than expected which may be an indicator for the acceptability of the MEP in the participating nursing homes. Indeed, after the conclusion of our intervention study, many of the 36 participating nursing homes continued the MEP and we received positive feedback from nursing home staff and participants. Furthermore, nursing staff received special training to continue the MEP following the intervention study. This training included information on how to adapt intensity level during exercise sessions when needed. Another strength of the study was the comprehensive acquisition of ADL performance using one proxybased questionnaire, and two performance-based assessments.

Nevertheless, our study has some limitations that must be considered when interpreting the results. First, we did not reach the intended sample size of 405 IWD because the number of participants that did not fulfill our inclusion criteria was higher than expected. However, a sensitivity analysis $\left(G^{*}\right.$ Power 3 , Version 3.1.9.2) showed that we were still able to detect small to medium effects. Second, our intervention program was initially designed with adjustments of exercise intensity during the 16-week intervention period. However, due to the high heterogeneity of individual characteristics of our study participants such as age or disease severity, we had to increase our safety efforts and could possibly not achieve an adequate training stimulus for all participants. Moreover, the theoretical recommendation of two training sessions per week (de Souto Barreto et al., 2016) was only partially feasible within our study. Hence, we strongly recommend including strategies to support adherence as proposed by van der Wardt et al. (2017). Another factor that may limit our findings is that we did not control for any other interventions that might have taken place during the conduct of our study in the IG no CG. Therefore, we cannot rule out that other social or ther- 
apeutic interventions may have biased our results. Another limitation is related to the number of incomplete data sets which reduced the sample size for statistical analysis in the per protocol analysis. Especially the BI as a proxy-based measurement filled in by nursing home staff was less often completed than the other assessments carried out by our study staff. Other reasons for incomplete data sets may be daily form and mood, depressive symptoms, other severe impairments or schedule conflicts. As we did not assess depressive symptoms with an evaluated assessment tool, we were not able to make assumptions about the possible influence of depression on ADL performance. Finally, our study did not include a followup assessment or a long-term monitoring of the continuation of the MEP in the participating nursing homes, so we do not have any results about the longterm effects of our intervention.

\section{Conclusion}

Our study showed that a one-size-fits-all MEP is not effective in improving ADL performance in a heterogeneous group of IWD. Nevertheless, improving or maintaining ADL performance of IWD living in nursing homes is critically important, as it is related to a better quality of life and a reduced care effort for nursing home staff. According to our responder analysis, particularly IWD with poor baseline motor performance responded positively to our exercise program, implying an insufficient exercise intensity for participants with better baseline values. Cognitive function and motor performance explained up to $51.4 \%$ of ADL performance with more motor than cognitive assessments as significant cofounders.

Our results are relevant for further research as they underline the need for individualized MEP for IWD, and a more critical handling with theoretical recommendations transferred into practice. Given the lack of intensity adjustments during our intervention period, future PE interventions should consider monitoring the progression of exercise intensity more precisely. In line with the concept of individualized medicine, further intervention studies should consider the individ- ual cognitive and motor impairments of IWD at baseline or at regular times of an intervention program to customize PE intervention content. Furthermore, future studies should account for the high heterogeneity in this target group with regard to disease severity, or cognitive function and motor performance status, and identify more homogeneous subgroups, e.g., by performing a cluster analysis. Using mobile health applications may help to indicate individual deficits (e.g., in balance, gait performance) at baseline and may therefore, present an effective and efficient solution for individually tailoring exercise interventions. However, this may only be applicable in small training groups or one-on-one guided training sessions. Further studies should investigate the usability and feasibility of mobile health applications for implementing individual exercise in nursing homes. Finally, studies comparing the effects of an individualized PE intervention on ADL performance, compared to a general PE intervention and a control group, are warranted.

\section{Corresponding address

Jelena Bezold
Institute of Sports and Sports
Science, Karlsruhe Institute of
Technology
Karlsruhe, Germany
Jelena.bezold@kit.edu

Funding. This project is financially supported by the Dietmar Hopp Stiftung (St. Leon-Roth, Germany). The sponsor does not have any role in the design of the study, neither in its execution, the data collection, or the analysis and interpretation of the data.

Author Contribution. All authors contributed to the study conception, design, and implementation of the study. J. Bezold, S. Trautwein, and B. Barisch-Fritz analyzed the data. J. Bezold interpreted the data. J. Bezold wrote the first draft of the manuscript and all authors provided critical feedback on previous versions of the manuscript. All authors approved the final manuscript.

Funding. Open Access funding enabled and organized by Projekt DEAL.

\section{Declarations}

Conflict of interest. J. Bezold, S. Trautwein, B. BarischFritz, A. Scharpf, J. Krell-Roesch, C. R. Nigg and A. Woll declare that they have no competing interests.
All procedures performed in studies involving human participants or on human tissue were in accordance with the ethical standards of the institutional (ethics committee of the Karlsruhe Institute of Technology, Karlsruhe, Germany) and/or national research committee and with the 1975 Helsinki declaration and its later amendments or comparable ethical standards. Informed consent was obtained from all individual participants included in the study.

Open Access. This article is licensed under a Creative Commons Attribution 4.0 International License, which permits use, sharing, adaptation, distribution and reproduction in any medium or format, as long as you give appropriate credit to the original author(s) and the source, provide a link to the Creative Commons licence, and indicate if changes were made. The images or other third party material in this article are included in the article's Creative Commons licence, unless indicated otherwise in a credit line to the material. If material is not included in the article's Creative Commons licence and your intended use is not permitted by statutory regulation or exceeds the permitted use, you will need to obtain permission directly from the copyright holder. To view a copy of this licence, visit http://creativecommons.org/licenses/by/4.0/.

\section{References}

Barisch-Fritz, B., Barisch, M., Trautwein, S., Scharpf, A., Bezold, J., \& Woll, A. (2020). Designing a mobile app for treating individuals with dementia: combining ux research with sports science. In M. Lames, A. Danilov, E. Timme \& Y. Vassilevski (Eds.), Advances in Intelligent Systems and Computing. Proceedings of the 12th International Symposium on Computer Science in Sport (IACSS 2019) (Vol. 1028, pp. 185-192). Berlin Heidelberg: Springer. https://doi.org/10. 1007/978-3-030-35048-2 22.

Barisch-Fritz, B., Trautwein, S., Scharpf, A., KrellRoesch, J., \& Woll, A. (2021). Effects of a 16week multimodal exercise program on physical performance in individuals with dementia: a multicenter randomized controlled trial. Journal of Geriatric Physical Therapy. https://doi.org/10. 1519/JPT.0000000000000308.

Blankevoort, C. G., van Heuvelen, M. J.G., \& Scherder, E. J.A. (2013). Reliability of six physical performance tests in older people with dementia. Physical Therapy, 93(1), 69-78. https://doi.org/ 10.2522/ptj.20110164.

Bossers, W.J.R., van der Woude, L.H.V., Boersma, F., Hortobágyi, T., Scherder, E.J.A., \& van Heuvelen, M. J.G. (2016). Comparison of effect of two exercise programs on activities of daily living in individuals with dementia: a 9-week randomized, controlled trial. Journal of the American Geriatrics Society, 64(6), 1258-1266. https://doi.org/10.1111/jgs.14160.

Boyd, P. A., Wilks, S. E., \& Geiger, J. R. (2018). Activities of daily living assessment among nursing home residents with advanced dementia: psychometric reevaluation of the bristol activities of daily living scale. Health \& Social Work, 43(2), 101-108. https://doi.org/10.1093/hsw/hly010.

Bürge, E., Berchtold, A., Maupetit, C., Bourquin, N. M.P., von Gunten, A., Ducraux, D., Zumbach, S., Peeters, A., \& Kuhne, N. (2017). Does physical exercise improve adl capacities in people over 65 years with moderate or severe dementia hospitalized in an acute psychiatric setting? A multisite randomized clinical trial. International 
Psychogeriatrics, 29(2), 323-332. https://doi. org/10.1017/S1041610216001460.

Farrell, M. K., Rutt, R. A., Lusardi, M. M., \& Williams, A. K (2010). Reliability of the physical performance test in people with dementia. Physical \& Occupational Therapy in Geriatrics, 28(2), 144-153. https://doi.org/10.3109/02703181. 2010.487973.

Faul, F., Erdfelder, E., Lang, A.-G., \& Buchner, A. (2007). G* power 3: A flexible statistical power analysis program for the social, behavioral, and biomedical sciences. Behavior Research Methods, 39(2), 175-191. https://doi.org/10. 3758/bf03193146.

Folstein, M. F., Folstein, S. E., \& McHugh, P. R. (1975). "Mini-mental state". A practical method for grading the cognitive state of patients for the clinician. Journal of Psychiatric Research, 12(3), 189-198.

Forbes, D., Forbes, S.C., Blake, C. M., Thiessen, E. J., \& Forbes, S. (2015). Exercise programs for people with dementia. The Cochrane Database of Systematic Reviews. https://doi.org/10.1002/ 14651858.CD006489.pub4.

Garcia-Pinillos, F., Cozar-Barba, M., Munoz-Jimenez, M., Soto-Hermoso, V., \& Latorre-Roman, P. (2016). Gait speed in older people: an easy test for detecting cognitive impairment, functional independence, and health state. Psychogeriatrics, 16(3), 165-171. https://doi. org/10.1111/psyg.12133.

Giebel, C. M., Sutcliffe, C., \& Challis, D. (2015). Activities of daily living and quality of life across different stages of dementia: a UK study. Aging \& Mental Health, 19(1), 63-71. https://doi.org/10.1080/ 13607863.2014.915920.

Graessel, E., Viegas, R., Stemmer, R., Küchly, B., Kornhuber, J., \& Donath, C. (2009). The erlangen test of activities of daily living: first results on reliability and validity of a short performance test to measure fundamental activities of daily living in dementia patients. International Psychogeriatrics, 21(1), 103-112. https://doi. org/10.1017/S1041610208007710.

Graham, J.E., Ostir, G. V., Kuo, Y.-F., Fisher, S.R., \& Ottenbacher, K. J. (2008). Relationship between test methodology and mean velocity in timed walk tests: a review. Archives of Physical Medicine and Rehabilitation, 89(5), 865-872. https://doi. org/10.1016/j.apmr.2007.11.029.

Guralnik, J. M., Simonsick, E. M., Ferrucci, L., Glynn, R. J., Berkman, L. F., Blazer, D. G., Scherr, P. A., \&Wallace, R. B. (1994). A short physical performancebattery assessing lower extremity function: association with self-reported disability and prediction of mortality and nursing home admission. Journal of Gerontology, 49(2), M85-M94. https://doi. org/10.1093/geronj/49.2.m85.

Hagovska, M., \& Nagyova, I. (2017). The transfer of skills from cognitive and physical training to activities of daily living: a randomised controlled study. European Journal of Ageing, 14(2), 133-142. https://doi.org/10.1007/s10433-016-0395-y.

Hampel, H., O'Bryant, S.E., Durrleman, S., Younesi, E., Rojkova, K., Escott-Price, V., Corvol, J.-C., Broich, K., Dubois, B., \& Lista, S. (2017). A precision medicine initiative for alzheimer's disease: The road ahead to biomarker-guided integrative disease modeling. Climacteric, 20(2), 107-118. https://doi.org/10.1080/13697137 2017.1287866

Helbostad, J. L., Vereijken, B., Becker, C., Todd, C., Taraldsen, K., Pijnappels, M., Aminian, K., \& Mellone, S. (2017). Mobile health applications to promote active and healthy ageing. Sensors. https://doi.org/10.3390/s17030622.

Henry-Sánchez, J.T., Kurichi, J.E., Xie, D., Pan, Q., \& Stineman, M.G.(2012). Doelderlypeopleatmore severe activity of daily living limitation stages fall more? American Journal of Physical Medicine \& Rehabilitation, 91(7), 601-610. https://doi.org/ 10.1097/PHM.0b013e31825596af.

Henskens, M., Nauta, I. M., Drost, K. T., \& Scherder, E. J. (2018). The effects of movement stimulation on activities of daily living performance and quality of life in nursing home residents with dementia: a randomized controlled trial. Clinical Interventions in Aging, 13, 805-817. https://doi. org/10.2147/CIA.S160031.

Johansen, R. H., Olsen, K., Bergh, S., Benth, J. Š., Selbæk, G., \& Helvik, A.-S. (2020). Course of activities of daily living in nursing home residents with dementia from admission to 36-month followup. BMC Geriatrics, 20(1), 488. https://doi.org/10 1186/s12877-020-01877-1.

Lam, F. M., Huang, M.-Z., Liao, L.-R., Chung, R. C., Kwok, T.C., \& Pang, M.Y. (2018). Physical exercise improves strength, balance, mobility, and endurance in people with cognitive impairment and dementia: A systematic review. Journal of Physiotherapy, 64(1), 4-15. https://doi.org/10. 1016/j.jphys.2017.12.001.

Lamb, S.E., Sheehan, B., Atherton, N., Nichols, V., Collins, H., Mistry, D., Dosanjh, S., Slowther, A. M. Khan, I., Petrou, S., \& Lall, R. (2018). Dementia and physical activity (dapa) trial of moderate to high intensity exercise training for people with dementia: randomised controlled trial. BMJ, 361, k1675. https://doi.org/10.1136/bmj.k1675.

Littbrand, H., Lundin-Olsson, L., Gustafson, Y., \& Rosendahl, E. (2009). The effect of a high-intensity functional exercise program on activities of daily living: A randomized controlled trial in residential care facilities. Journal of the American Geriatrics Society, 57(10), 1741-1749. https:// doi.org/10.1111/j.1532-5415.2009.02442.x.

Littbrand, H., Stenvall, M., \& Rosendahl, E. (2011). Applicability and effects of physical exercise on physical and cognitive functions and activities of daily living among people with dementia: a systematic review. American Journal of Physical Medicine \& Rehabilitation, 90(6), 495-518. https://doi.org/10.1097/PHM 0b013e318214de26.

Luttenberger, K., Schmiedeberg, A., \& Gräßel, E. (2012). Activities of daily living in dementia: Revalidation of the E-ADL Test and suggestions for further development. BMC Psychiatry, 12, 208. https://doi.org/10.1186/1471-244X-12208.

Mahoney, F.I., \& Barthel, D.W. (1965). Functional evaluation: the barthel index. Maryland State Medical Journal, 14,61-65.

Martyr, A., \& Clare, L. (2012). Executive function and activities of daily living in alzheimer's disease: a correlational meta-analysis. Dementia and Geriatric Cognitive Disorders, 33(2-3), 189-203. https://doi.org/10.1159/000338233.

Miyamoto, Y., Tachimori, H., \& Ito, H. (2010). Formal caregiver burden in dementia: Impact of behavioral and psychological symptoms of dementia and activities of daily living. Geriatric Nursing, 31(4), 246-253. https://doi.org/10. 1016/j.gerinurse.2010.01.002

Mlinac, M.E., \& Feng, M.C. (2016). Assessment of activities of daily living, self-care, and independence. Archives of Clinical Neuropsychology,
31(6), 506-516. https://doi.org/10.1093/arclin/ acw049.

Müllers, P., Taubert, M., \& Müller, N.G. (2019). Physical exercise as personalized medicine for dementia prevention? Frontiers in Physiology, 10, 672. https://doi.org/10.3389/fphys.2019.00672.

Nickel, F., Barth, J., \& Kolominsky-Rabas, P.L. (2018). Health economic evaluations of nonpharmacological interventions for persons with dementia and their informal caregivers: $A$ systematic review. BMC Geriatrics, 18(1), 69. https://doi.org/10.1186/s12877-018-0751-1.

Patterson, C. (2018). World Alzheimer Report 2018-The state of the art of dementia research: New frontiers. London. https://www.alzint.org/ u/WorldAlzheimerReport2018.pdf. Accessed 10 Feb 2021.

Pickett, J., Bird, C., Ballard, C., Banerjee, S., Brayne C., Cowan, K., Clare, L., Comas-Herrera, A., Corner, L., Daley, S., Knapp, M., Lafortune, L., Livingston, G., Manthorpe, J., Marchant, N., Moriarty, J., Robinson, L., van Lynden, C., Windle, G., \& Walton, C. (2018). A roadmap to advance dementia research in prevention, diagnosis, intervention, and care by 2025 . International Journal of Geriatric Psychiatry, 33(7), 900-906. https://doi.org/10.1002/gps.4868

Podsiadlo, D., \& Richardson, S. (1991). The timed "up \& go": a test of basic functional mobility for frail elderly persons. Journal of the American Geriatrics Society, 39(2), 142-148. https://doi. org/10.1111/j.1532-5415.1991.tb01616.x.

Portegijs, E., Rantakokko, M., Viljanen, A., Sipilä, S., \& Rantanen, T. (2016). Identification of older people at risk of adl disability using the life-space assessment: a longitudinal cohort study. Journal of the American Medical Directors Association, 17(5), 410-414. https://doi.org/10. 1016/j.jamda.2015.12.010.

Prizer, L. P., \& Zimmerman, S. (2018). Progressive support for activities of daily living for persons living with dementia. The Gerontologist, 58(suppl_1), S74-S87. https://doi.org/10.1093/ geront/gnx103.

Reitan, R. M. (1958). Validity of the trail making test as an indicator of organic brain damage. Perceptual and Motor Skills, 8(3), 271-276. https://doi.org/ 10.2466/pms.1958.8.3.271.

Reuben, D. B., \& Siu, A. L. (1990). An objective measure of physical function of elderly outpatients. The physical performance test. Journal of the American Geriatrics Society, 38(10), 1105-1112.

Rolland, Y., Pillard, F., Klapouszczak, A., Reynish, E., Thomas, D., Andrieu, S., Rivière, D., \& Vellas, B. (2007). Exercise program for nursing home residents with alzheimer's disease: a 1-year randomized, controlled trial. Journal of the American Geriatrics Society, 55(2), 158-165. https://doi.org/10.1111/j.1532-5415. 2007.01035.x.

Rossiter-Fornoff, J.E., Wolf, S. L., Wolfson, L.I., \& Buchner, D.M. (1995). A cross-sectional validation study of the ficsit common data base static balance measures. Frailty and injuries: cooperative studies of intervention techniques. The Journals of Gerontology. Series A, Biological Sciences and Medical Sciences, 50(6), M291-M297.

Saghaei, M., \& Saghaei, S. (2011). Implementation of an open-source customizable minimization program for allocation of patients to parallel groups in clinical trials. Journal of Biomedical Science and Engineering, 04(11), 734-739. https://doi.org/10.4236/jbise.2011.411090. 
Sanders, L. M. J., Hortobágyi, T., Karssemeijer, E. G. A., Van der Zee, E.A., Scherder, E.J.A., \& van Heuvelen, M.J.G. (2020). Effects of low- and high-intensity physical exercise on physical and cognitive function in older adults with dementia: a randomized controlled trial. Alzheimer's Research \& Therapy. https://doi.org/10.1186/ s13195-020-00597-3.

Scharpf, A., Servay, S., \& Woll, A. (2013). Auswirkungen von körperlicher Aktivität auf demenzielle Erkrankungen. Sportwissenschaft, 43(3), 166-180. https://doi.org/10.1007/s12662-0130295-7.

Shulman, K. I., Shedletsky, R., \& Silver, I. L. (1986). The challenge of time: Clock-drawing and cognitive function in the elderly. International Journal of Geriatric Psychiatry, 1(2), 135-140. https://doi. org/10.1002/gps.930010209.

Sköldunger, A., Wimo, A., Sjögren, K., Björk, S., Backman, A., Sandman, P.-O., \& Edvardsson, D. (2019). Resource use and its association to cognitive impairment, adl functions, and behavior in residents of swedish nursing homes: Results from the U-age program (swenis study). International Journal of Geriatric Psychiatry, 34(1), 130-136. https://doi.org/10. 1002/gps.5000.

Sopina, E., \& Sørensen, J. (2018). Decision modelling of non-pharmacological interventions for individuals with dementia: a systematic review of methodologies. Health Economics Review, 8(1), 8. https://doi.org/10.1186/s13561-0180192-8.

de Souto Barreto, P., Morley, J.E., Chodzko-Zajko, W., Pitkala, K.H., Weening-Djiksterhuis, E., Rodriguez-Mañas, L., Barbagallo, M., Rosendahl E., Sinclair, A., Landi, F., Izquierdo, M., Vellas, B., \& Rolland, Y. (2016). Recommendations on physical activity and exercise for older adults living in long-term care facilities: a taskforce report. Journal of the American Medical Directors Association, 17(5), 381-392. https://doi.org/10. 1016/j.jamda.2016.01.021.

Sun, M., Mainland, B. J., Ornstein, T.J., Sin, L.G., \& Herrmann, N. (2018). Correlates of nursing care burden among institutionalized patients with dementia. International Psychogeriatrics, 30(10), 1549-1555. https://doi.org/10.1017/ S104161021800025X.

Telenius, E.W., Engedal, K., \& Bergland, A. (2013). Physical performance and quality of life of nursing-home residents with mild and moderate dementia. International Journal of Environmental Research and Public Health, 10(12), 6672-6686. https://doi.org/10.3390/ ijerph10126672.

Thurm, F., Scharpf, A., Liebermann, N., Kolassa, S., Elbert, T., Lüchtenberg, D., Woll, A., \& Kolassa, I.-T. (2011). Improvement of cognitive function after physical movement training in institutionalized very frail older adults with dementia. GeroPsych 24(4), 197-208. https://doi.org/10.1024/16629647/a000048.

Toots, A., Littbrand, H., Lindelöf, N., Wiklund, R., Holmberg, H., Nordström, P., Lundin-Olsson, L., Gustafson, Y., \& Rosendahl, E. (2016). Effects of a high-intensity functional exercise program on dependence in activities of daily living and balance in older adults with dementia. Journal of the American Geriatrics Society, 64(1), 55-64. https://doi.org/10.1111/jgs.13880.

Trautwein, S., Barisch-Fritz, B., Scharpf, A., Bossers, W., Meinzer, M., Steib, S., Stein, T., Bös, K., Stahn, A., Niessner, C., Altmann, S., Wittelsberger,
R. \& Woll, A. (2019). Recommendations for assessing motor performance in individuals with dementia: suggestions of an expert panel-a qualitative approach. European Review of Aging and Physical Activity, 16, 5. https://doi.org/10.1186/s11556-019-0212-7.

Trautwein, S., Barisch-Fritz, B., Scharpf, A., Ringhof, S. Stein, T., Krell-Roesch, J., \& Woll, A. (2020). Effects of a 16-week multimodal exercise program on gait performance in individuals with dementia: a multicenter randomized controlled trial. $B M C$ Geriatrics, 20(1), 245. https://doi.org/10.1186/ s12877-020-01635-3.

Trautwein, S., Scharpf, A., Barisch-Fritz, B., Niermann C., \& Woll, A. (2017). Effectiveness of a 16-week multimodal exercise program on individuals with dementia: study protocol for a multicente randomized controlled trial. JMIR Research Protocols, 6(3), e35. https://doi.org/10.2196/ resprot.6792.

van der Wardt, V., Hancox, J., Gondek, D., Logan, P. d Nair, R., Pollock, K., \& Harwood, R. (2017). Adherence support strategies for exercise interventions in people with mild cognitive impairment and dementia: A systematic review. Preventive Medicine Reports, 7, 38-45. https:// doi.org/10.1016/j.pmedr.2017.05.007.

Wilde, N.J., Strauss, E., \& Tulsky, D.S. (2004). Memory span on the wechsler scales. Journal of Clinical and Experimental Neuropsychology, 26(4), 539-549. https://doi.org/10.1080/ 13803390490496605.

World Health Organization, \& Alzheimer's Disease International (2012). Dementia: a public health priority. Geneva: Word Health Organization.

Yi, Y., Ding, L., Wen, H., Wu, J., Makimoto, K., \& Liao, X. (2020). Is barthel index suitable for assessing activities of daily living in patients with dementia? Frontiers in Psychiatry, 11, 282. https://doi.org/10.3389/fpsyt.2020.00282.

Yu, F., Salisbury, D., \& Mathiason, M. A. (2021). Interindividual differences in the responses to aerobic exercise in alzheimer's disease: findings from the fit-ad trial. Journal of Sport and Health Science, 10(1), 65-72. https://doi.org/10.1016/j.jshs. 2020.05 .007$. 